
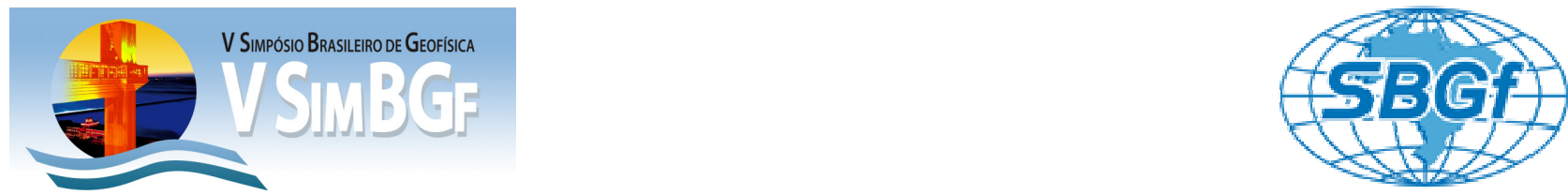

\title{
Uso de AVO para a identificação de selos: Um estudo de caso da Bacia do Parnaíba.
}

Bruno Rodrigues de Carvalho, Invision Geophysics

Aisgo Oguro, OGX Petróleo SA

Roberto Baldanza Ribeiro, OGX Petróleo SA

Copyright 2012, SBGf - Sociedade Brasileira de Geofísica

Este texto foi preparado para a apresentação no V Simpósio Brasileiro de Geofísica, Salvador, 27 a 29 de novembro de 2012. Seu conteúdo foi revisado pelo Comitê Técnico do V SimBGf, mas não necessariamente representa a opinião da SBGf ou de seus associados. É proibida a reprodução total ou parcial deste material para propósitos comerciais sem prévia autorização da SBGt.

\section{Abstract}

In this work we demonstrate how the attributes of AVO (amplitude versus offset) can be used in the exploratory cycle Brazilian onshore sedimentary basins. Although the most common use of AVO studies focus on defining the reservoir and its fluid content, the signing of the seal rock in certain basins have equal importance. Due to the fact that traditionally the quality of land seismic data in Brazil imposed a limitation to the use of this technique, we designed a careful preconditioning flow and quality control data, thus enabling the derivation of attributes and AVO analysis.

Using AVO attributes initially and after elastic inversion, it was possible to improve the knowledge of the basaltic sills Parnaíba Basin, which are considered a major regional seal. The cases analyzed show that regions where the seal is of good quality has differentiated AVO signature.

Two seismic lines and two wells were used to compose analysis, first through a well with high gas saturation and the other across a pit occurrence of accumulation. One possible explanation for the dry well is the lack of seal or stamp revenue due transpressive efforts.

\section{Introdução}

A Bacia do Parnaíba está localizada na porção noroeste da Região Nordeste do Brasil; abrange área de cerca de $600.000 \mathrm{~km}^{2}$ e engloba grandes áreas dos estados do Maranhão e do Piauí, partes dos estados do Pará e do Tocantins (Figura 1) e, ainda pequenas partes dos estados do Ceará e da Bahia (Della Fávera, 1990). Os folhelhos da Fm. Pimenteiras são considerados os principais geradores da Bacia do Parnaíba. Essas rochas foram depositadas durante um evento anóxico ocorrido no Devoniano (Rodrigues, 1995). São também considerados geradores potencias os folhelhos da Fm. Tianguá (Siluriano) e Longá (Devoniano-Fameniano) (ANP, 2002). A migração foi facilitada pela ocorrência de falhas decorrentes dos efeitos da orogenia Eoherciniana e uma fase tectônica tardia, ocorrida durante o evento transpressivo do Cretáceo, representado por falhas de perfil reverso. A eficiência da migração foi comprovada por poços na área, nos arenitos da Fm. Poti, sotopostos à espessa soleira de diabásio intrudida nos folhelhos da Fm. Pimenteiras. Também ocorre espessa zona de gás em arenitos da Fm. Cabeças. O trapeamento dos reservatórios principais portadores de gás, na área em questão, consiste em uma estrutura quaquaversal. Esse trapeamento foi formado por esforços orogênicos no eocarbonífero. Os falhamentos originados durante esse evento foram posteriormente reativados como falhas de perfil reverso, durante 0 evento transpressivo do Cretáceo. As espessas intrusões de diábasio formam o principal selo para os arenitos da Fm Poti, portanto o imageamento melhorado destes refletores na sísmica, torna-se imprescindível para identificação de futuros leads na bacia. Para os reservatórios portadores de gás da Fm. Cabeças, o selo efetivo são os folhelhos da Fm. Longá (Vaz et al., 2007). Admite-se que as intrusões ígneas de diabásio ocorreram concomitantemente com evento tectônico que propiciou a formação de trapas estruturais e a geração e migração de hidrocarbonetos. Tectonismos posteriores remobilizariam hidrocarbonetos já trapeados e formariam trapas para eventual geração tardia de hidrocarboneto.

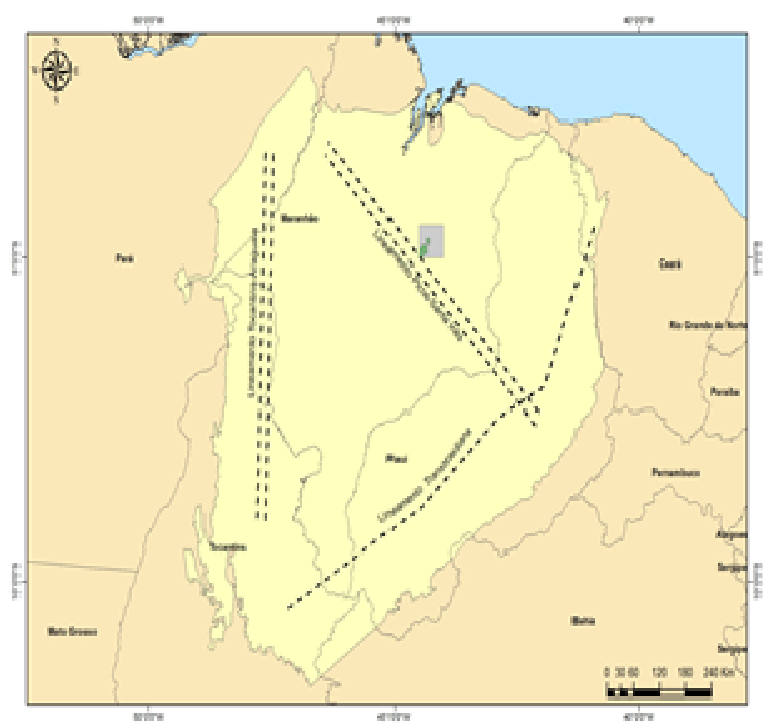

Figura 1 - Localização geográfica da Bacia do Parnaíba (em amarelo), com destaque para os lineamentos TocantinsAraguaia a oeste da bacia, Transbrasiliana ao sul e Picos-Santa Inês a leste (linhas pontilhadas em preto).

\section{Metodologia/ Problema Investigado}

Neste trabalho, um melhor imageamento das soleiras de diabásio tornou-se necessário, pois além dos requerimentos de qualidade do dado para inversão, o condicionamento espectral com o aumento de resolução, 
facilita ao intérprete, o mapeamento das trapas, selos, e reservatórios deste sistema. Para tanto, o fluxo de condicionamento utilizado foi: moveout residual para alinhamento das reflexões no domínio do CMP; correção dos efeitos de absorção e dispersão para aumento do conteúdo de frequência do sinal; filtragem de ruídos aleatórios usando a transformada de curvlet (Braga, 2011).

O método adotado para inversão das linhas $2 \mathrm{D}$ foi a aproximação de Aki \& Richards (1980) para as equações de Knoot-Zoeppritz, dos coeficientes de reflexão da onda plana.

\section{Resultados}

Com o condicionamento dos gathers, foi obtido um melhor imageamento das seções sísmicas empilhadas, em ambas as linhas 2D estudadas (Figura 3). Este tratamento proporcionou o aumento do conteúdo de frequência pela aplicação da correção dos efeitos de absorção e dispersão e o aumento da razão sinal/ruído pela diminuição do conteúdo de ruído aleatório, melhorando consideravelmente, o comportamento da amplitude ao longo dos offsets (Figura 2). Este tratamento dos gathers foi imprescindível para a geração dos atributos elásticos Intercept, Gradient e refletividade da onda compressional ( $R p)$, uma vez que o conteúdo de ruído influencia negativamente a inversão (Gray and Andersen, 2000).

A figura 2 a mostra o poço $\mathrm{H}$ utilizado para modelagem do sintético e a respectiva wavelet convolvida no processo. A wavelet utilizada foi uma Ricker com freqüência de 35 $\mathrm{Hz}$ e fase zero. A figura 2c mostra a comparação entre $\mathrm{O}$ sintético e dado sem condicionamento. O dado CMP original apresenta baixa razão sinal/ruído e sem alinhamento dos refletores. A figura $2 b$ compara 0 sintético com o dado já condicionado. O gather condicionado extraído ao longo do poço mostra boa amarração com o sintético, principalmente no objetivo analisado, conforme indicado pela Figura $2 \mathrm{~b}$ e $2 \mathrm{c}$. Com o condicionamento, foi possível observar entre os tempos 800 ms e 1100 ms, região do objetivo, uma boa distinção das camadas, fato que não era possível no dado original. $O$ dado sem condicionamento e empilhado (Figura 3) não apresenta boa qualidade no imageamento dos refletores, e conseqüentemente pouca continuidade nos corpos de diabásio dentro da região estudada.

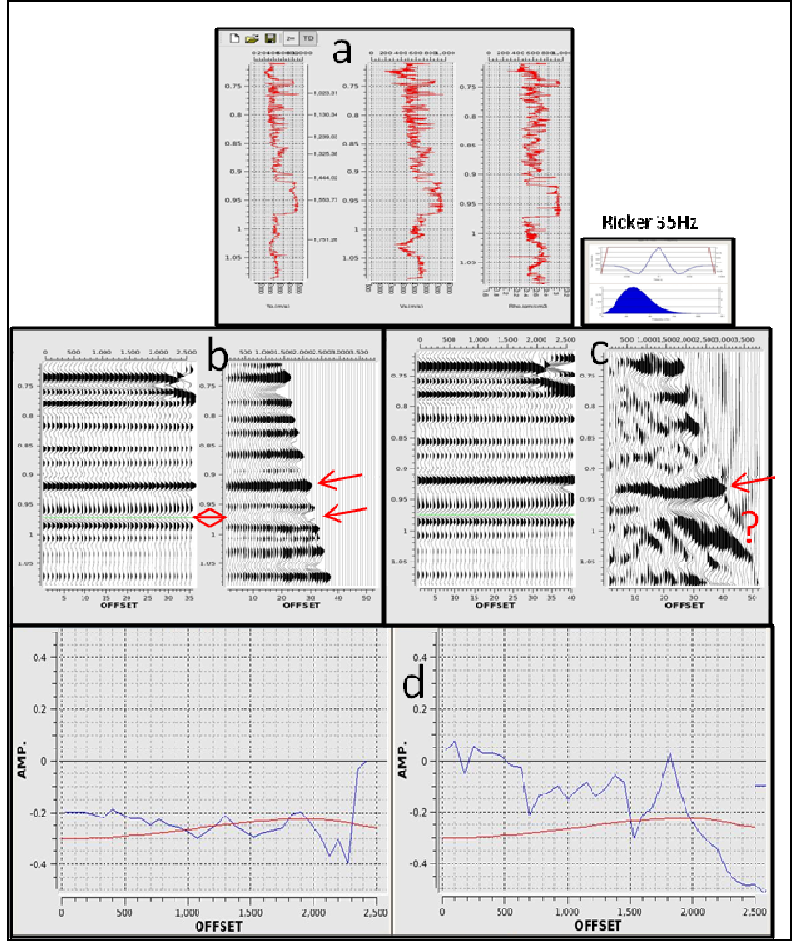

Figura 2 - (a) Representa o Poço h. Os perfis são respectivamente, velocidade compressional $(\mathrm{m} / \mathrm{s})$, cisalhante $(\mathrm{m} / \mathrm{s})$ e densidade $(\mathrm{g} / \mathrm{cm} 3)$. (b) sintético gerado apartir do poço $h$ com wavelet Ricker $35 \mathrm{~Hz}$ em fase zero comparado com o dado após a etapa de condicionamento sísmico. (c) o mesmo sintético da figura $2 b$ comparado com o dado sem condicionamento. (d) representa a análise de amplitude para o dado com

Analisando o crossplot de intercept $x$ gradient, verificouse que valores próximos à região correspondente a classe de AVO I (Castagna, et al.,1992), representavam a soleira de diabásio, nas seções sísmicas das duas linhas 2D (Figura 4). Entretanto, na linha S (poço seco), a delimitação do diabásio limitou-se à porção oeste da linha, não sendo mais identificado na região do poço. $\mathrm{Na}$ linha $\mathrm{H}$ (poço com hidrocarbonetos), a delimitação da soleira de diabásio a partir do crossplot, mostrou boa continuidade lateral na região do poço (Figura 4). 


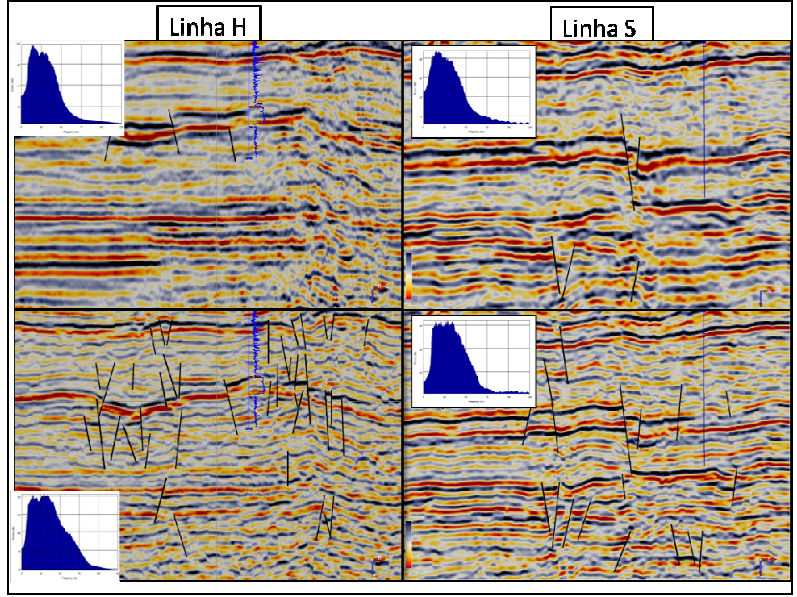

Figura 3 - Comparação entre as duas linhas 2-D, linhas $H$ e $S$. A linha $H$ com a presença de hidrocarboneto e linha $S$ sem a presença de hidrocarboneto. Na parte superior estão as linhas sem fluxo e inferior com fluxo. Tanto na linha $H$ quanto na linha $S$ observa-se uma boa melhoria tanto no imageamento quanto em seu conteúdo de freqüência.

Com a impedância compressional relativa, oriundo da inversão, o diabásio é representado nas duas linhas, por uma camada entre 800 e $1100 \mathrm{~ms}$ e de alta impedância (Figura 5). Seu limite inferior é coincidente com a entrada das areias, e é representada por uma camada de baixa impedância (Figura 5). Na linha $\mathrm{H}$ (cruzando poço com hidrocarbonetos), a soleira de diabásio apresenta boa continuidade lateral na região do poço, selando e trapeando as areias abaixo (Figura 5). $\mathrm{Na}$ linha $\mathrm{S}$ (cruzando poço seco), a soleira se faz presente na maior parte da seção, entretanto na região do poço, a mesma não é bem visualizada, apresentando valores menores de impedância comparada a região do diabásio circunvizinha ao poço, não apresentando continuidade lateral (Figura 5).

Outro evento importante da bacia que foi melhor imageado após a inversão, foi a deposição de sal correspondente as Fm. Motuca e Pedra de Fogo. Estes eventos são representados nas seções de impedância relativa integrado, como camadas de alta impedância, ocorrendo respectivamente em 600 e 680 ms, são contínuas ao longo das seções empilhadas e invertidas (Figura 5). Estes depósitos evaporíticos têm caráter regional, decorrente de evento de escala global ocorrido no final do Permiano, inicio do Triássico (Vaz et al., 2007).

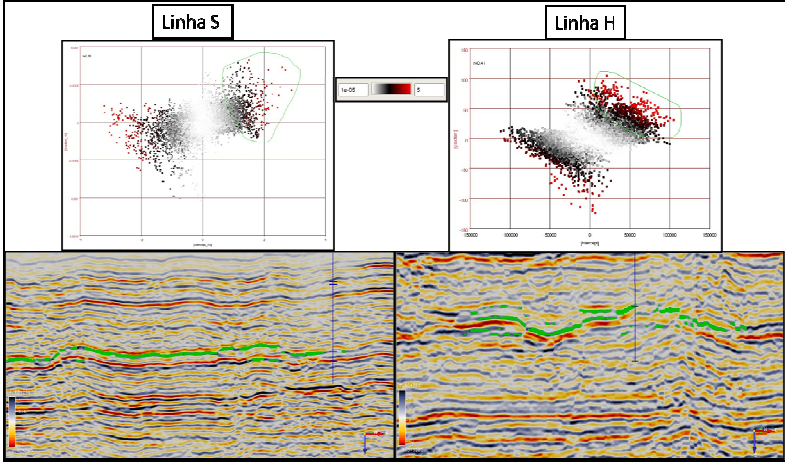

Figura 4 - O grafico de Intercept vs Gradiente na respectivas linhas $S$ e $H$. A escala de cor representa o atributo energia. Em destaque, em verde, a seção sísmica condicionada e a delimitação da soleira de diabásio

\section{Discussão e Conclusões}

A qualidade do imageamento sísmico é vital durante a campanha exploratória, assim como uma eventual caracterização do reservatório, tanto numa analise qualitativa, quanto quantitativa (Yilmaz, 2000). Após o aumento da resolução sísmica e a melhoria no controle da amplitude ao longo das interfaces sísmicas, foi possível uma interpretação mais segura dos principais refletores da bacia. A visualização das descontinuidades, decorrentes do regime de esforços trasnpressivos da área, também foram facilmente observadas ao longo das seções, fato este, dificultado nas seções sem tratamento (Figura 3). Na linha S contatou-se intenso falhamento na região do poço e proximidades. Estas descontinuidades possivelmente fraturaram a soleira de diabásio e permitiram a fuga do hidrocarboneto. Esta hipótese foi levantada, a partir da observação de perfis de imagem de poços análogos, que apresentavam fraturas no diabásio.

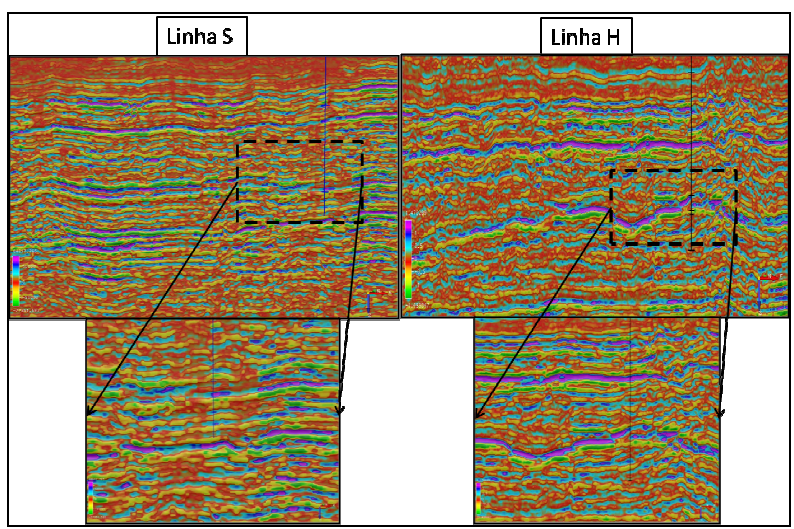

Figura 5 - Seções de impedância $P$ relativa das linhas $S$ e H. Em destaque, a soleira de diabásio representada por uma camada de alta impedância e contínua na região do poço $\mathrm{H}$, em contraste com a região do poço $S$. 


\section{Agradecimentos}

Agradecemos a OGX petróleo e gás por disponibilizar dados. O Marcos Amaral por incentivar a realização deste trabalho. Ao Paulo Cunha e João Caldeira por apresentar e propor os desafios da área de estudo. $\mathrm{O}$ Igor Braga pela ajuda e enriquecimento geofísico, a Tatiane Moura pelos esclarecimentos geológicos e Odilla Vilhena pela sua organização e seu comportamento altruísta. Agradecemos também a todos que ajudaram indiretamente a realização deste trabalho.

\section{Referências}

Aki, K. and Richards, P. G., 1980, Quantitative seismology: Theory and methods, v.1: W.H. Freeman and Co.

Della Fávera, J.C., 1990. Tempestitos da bacia do Parnaíba: um ensaio holístico. D.Sc. Thesis, Instituto de Geociências, Universidade Federal do Rio Grande do Sul, Porto Alegre. 243p.

Gray, F.D. and Andersen, E.A., 2000, The Application of AVO and Inversion to Formation Properties, World Oil, Vol. 221, No. 7.

Braga, I. L. S., 2011,Técnicas multiespectrais aplicada a fluxos de inversão e caracterização de reservatórios de hidrocarbonetos. PhD Thesis, Universidade Estadual do Norte Fluminense, Brazil (In Portuguese).

Rodrigues, R., 1995. A Geoquímica Orgânica na bacia do Parnaíba. D.Sc. Thesis, Instituto de Geociências, Universidade Federal do Rio Grande do Sul, Porto Alegre. 225p.

Rossetti, D. F., Truckenbrodt, W., Santos Junior, A. E., Clima do Cretáceo no Meio-Norte Brasileiro, 2001. In: ROSSETI, D. F., GOES, A. M., TRUCKENBRODT, W. (Ed.), 2001, O Cretáceo na Bacia de São Luis-Grajau. Belém: Museu Paraense Emilio Goeldi, p.67-76. Dynamics of Passive Margins. AGU Geodynamic Series, Washington (DC), USA, vol. 6: 147-153.

Silva, A. J. P., Lopes, R C., Vasconcelos. A. M., Bacias Sedimentares Paleozóicas e Meso-Cenozóicas Interiores. L. A. Bizzi, C. Schobbenhaus, R. M. Vidotti e J. H. Gonçalves (eds.) 2003. Geologia, Tectônica e Recursos Minerais do Brasil - CPRM Brasília, cap 2, p 55-85.

Vaz P. T., Rezende N. G. A. M., Joaquim Ribeiro Wanderley Filho J.R., Travassos W. A. S., maio/nov 2007, Boletim de Geociências da PETROBRAS, Rio de Janeiro, v. 15, n. 2, p. 253-263. 